\title{
Variables affecting smoothed particle hydrodynamics simulation of high velocity flyer plate impact experiments
}

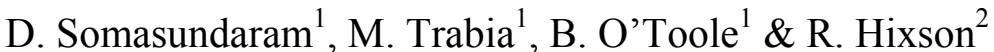 \\ ${ }^{1}$ Department of Mechanical Engineering, University of Nevada, USA \\ ${ }^{2}$ National Security Technologies, LLC, USA
}

\begin{abstract}
This paper describes our work to characterize the variables affecting the smoothed particle hydrodynamics (SPH) method in the LS-DYNA package for simulating high-velocity flyer plate impact experiments. LS-DYNA simulations are compared with one-dimensional experimental data of an oxygen-free highconductivity (OFHC) copper flyer plate impacting another plate of the same material and cross section at an impact velocity of $307 \mathrm{~m} / \mathrm{s}$. The comparison is made by measuring the velocity of a point on the back surface of the impact plate using the velocity interferometer system for any reflector (VISAR) technique. The Johnson-Cook material model is used in our simulations. Effects of particle density, bulk viscosity, specific heat parameter are studied and discussed.

Keywords: smoothed particle hydrodynamics, SPH, flyer plate experiment, LSDYNA, Johnson-Cook material model.
\end{abstract}

\section{Introduction}

Explosives or ballistic impacts result in large deformation, spallation, and accelerations as high as $500,000 \mathrm{G}$. In these cases, finite element methods may not be an effective modeling tool as the deformation of the elements become very distorted. A more suitable option is SPH, which is a relatively new meshfree Lagrangian-based numerical method developed specifically for solving these problems. This method does not require a numerical grid of elements to calculate the spatial derivative, which enables SPH method to avoid mesh tangling and distortion. In SPH, a set of particles represent the geometry. Each particle 
represents an interpolation point for which all properties are known. Nodal forces, energy and pressure are computed between each particle with a regular interpolation function known as the smoothing length. In addition to the smoothing length, LS-DYNA uses other variables to define the SPH model including, scale factor, particle approximation theory, initial number of neighbors, computational method for smoothing length initialization, bulk viscosity, and particle density.

Few researchers have considered the effects of particle density on SPH models. For example, Jackson and Fuchs [1] studied the effect of particle density on the simulation of a composite fuselage drop into water. Barsotti [2] also compared models using different particle densities for simulating a crushable foam aircraft arrestor bed. However, limited research has been done on characterizing how the other SPH variables, listed above, affect the behavior of an SPH model under dynamic conditions.

We seek to benchmark SPH capabilities by comparing simulation results with very fundamental experimental shock compression data. For this purpose, a onedimensional uniaxial strain experiment done previously by Hixson [3] is used. In this experiment, a stationary unrestricted target cylindrical plate $(38 \mathrm{~mm}$ diameter and $4 \mathrm{~mm}$ thick) is impacted by a flyer plate with the same cross section, but $1.78 \mathrm{~mm}$ thick, and at a velocity of $307 \mathrm{~m} / \mathrm{s}$ using a gas gun. Both plates are made of OFHC copper.

The free-surface velocity of the center point on the back surface of the target plate is measured using a Velocity Interferometer System for any Reflector (VISAR) technique as described by Barker and Hollenbach [4]. Figure 1 shows the measured velocity at the back surface of the target. This data set exhibits these three major characteristics: (1) elastic precursor wave, (2) plastic rise time, and (3) plateau. These three regions will be compared with various simulations with the objective of creating a model that reproduces these three characteristics closely. Later time (spall) data will not be considered in this work.

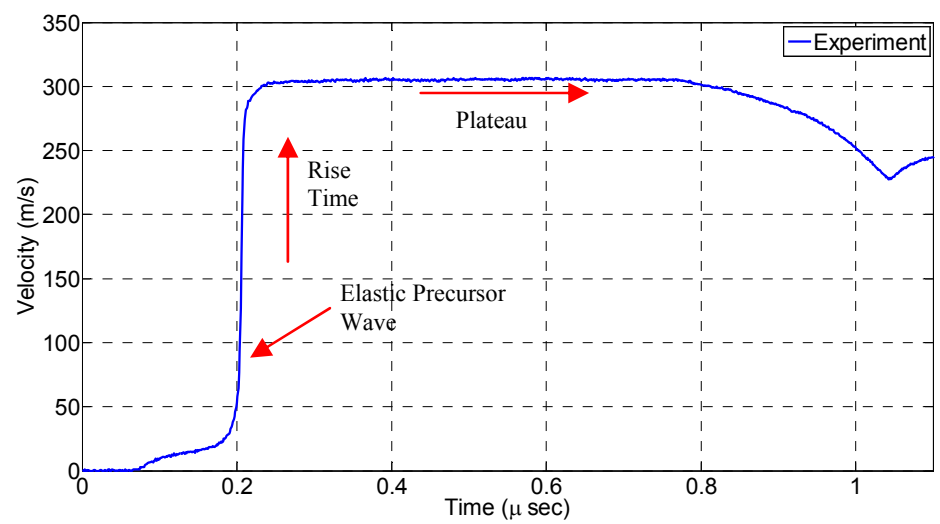

Figure 1: Measured velocity at the back surface of a one-dimensional uniaxial strain experiment. 


\section{Method}

The experiment described in Section 1 is modeled as a two-dimensional axisymmetric geometry in LS-DYNA (as shown in Figure 2). The Johnson-Cook material model (Johnson and Cook [5]) with Grüneisen equation of state (Hallquist [6]) is used for defining the material properties. In the Johnson-Cook material model the flow stress is expressed as

$$
\sigma_{\mathrm{y}}=\left(\mathrm{A}+\mathrm{B} \epsilon^{\mathrm{p}}\right)(1+\mathrm{C} \ln (\dot{\epsilon}))\left(1-\mathrm{T}^{* \mathrm{~m}}\right)
$$

where, $\sigma_{\mathrm{y}}$ is the flow stress, $\mathrm{A}$ is the yield stress under quasi-static conditions, $\mathrm{B}$ and $n$ are strain hardening parameters, $m$ is the temperature sensitivity and $C$ the strain rate dependence parameter. $\epsilon^{\mathrm{p}}$ is the effective plastic strain, and $\dot{\epsilon}$ is the effective total strain-rate normalized by quasi-static rate (ESPO) and defined as

$$
\dot{\epsilon}=\frac{\epsilon}{\text { EPSO }} \text {. }
$$

$\mathrm{T}^{*}$ is the homologous temperature defined by the relation

$$
T^{*}=\frac{\mathrm{T}-\mathrm{T}_{\mathrm{r}}}{\mathrm{T}_{\mathrm{m}}-\mathrm{T}_{\mathrm{r}}}
$$

where $T_{r}$ and $T_{m}$ are the reference temperature and melting temperature, respectively. The temperature is calculated using the equation [7]:

$$
\Delta \mathrm{T}=\frac{1}{\rho \mathrm{C}_{\mathrm{v}}} \int \sigma \mathrm{d} \epsilon^{\mathrm{p}},
$$

where $\Delta \mathrm{T}$ is the change in temperature, $\rho$ is the density and $\mathrm{C}_{\mathrm{v}}$ is the specific heat parameter.

In LS-DYNA, the Grüneisen equation of state is expressed as

$$
P=\frac{\rho_{0} C^{2} \mu\left[1+\left(1-\frac{\gamma_{0}}{2}\right) \mu-\frac{a}{2} \mu^{2}\right]}{\left[1-\left(S_{1}-1\right) \mu-S_{2} \frac{\mu^{2}}{\mu+1}-S_{3} \frac{\mu^{3}}{(\mu+1)^{2}}\right]^{2}}+\left(\gamma_{0}+a \mu\right) E,
$$

where $\mathrm{P}$ is the pressure, $\mathrm{S}_{1}, \mathrm{~S}_{2}, \mathrm{~S}_{3}$ are the coefficients of slope of shock and particle velocity curve, $\Gamma$ is Grüneisen coefficient, $a$ is the volume correction factor, $\rho$ is the density $\mathrm{C}$ is the Hugoniot intercept of the metal, and $\mu=\left(\rho / \rho_{0}\right)-1$.

The parameters of the model, are taken from Johnson and Cook [5] and Marsh [7] with slight changes, and are listed in Tables 1 and 2. The effects of varying the three SPH variables are studied: bulk viscosity, specific heat capacity, and mesh density. Preliminary simulation results showed that varying parameters like particle approximation theory, initial number of neighbors, and computational method for smoothing length initialization did not affect the results. 


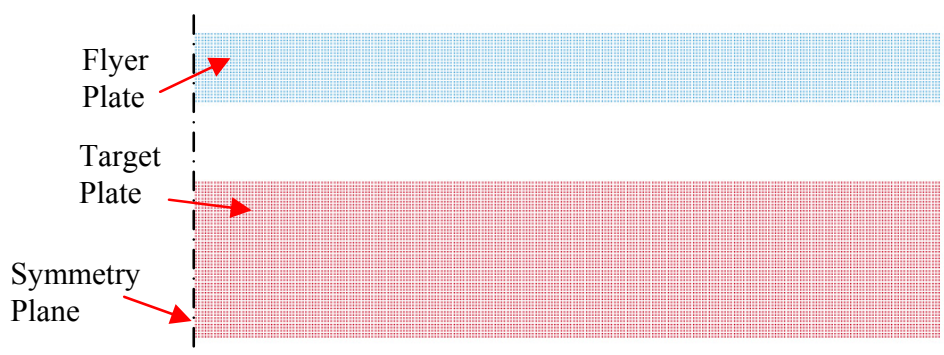

Figure 2: Two-dimensional axisymmetric model in LS-DYNA.

Table 1: Johnson-Cook parameter.

\begin{tabular}{|c|c|c|c|c|c|c|c|c|}
\hline $\begin{array}{c}\mathrm{A} \\
(\mathrm{MPa})\end{array}$ & $\begin{array}{c}\mathrm{B} \\
(\mathrm{MPa})\end{array}$ & $\mathrm{C}$ & $\mathrm{N}$ & $\mathrm{m}$ & $\begin{array}{c}\varepsilon_{0} \\
(1 / \mathrm{s})\end{array}$ & $\begin{array}{c}\mathrm{T}_{\mathrm{m}} \\
(\mathrm{K})\end{array}$ & $\begin{array}{c}\mathrm{C}_{\mathrm{v}} \\
(\mathrm{J} / \mathrm{kg} \mathrm{k})\end{array}$ & $\begin{array}{c}\text { Spall } \\
(\mathrm{GPa})\end{array}$ \\
\hline 89.7 & 292 & 0.31 & 0.025 & 1.09 & 1 & 1381 & 392 & 1.79 \\
\hline
\end{tabular}

Table 2: Grüneisen equation of state.

\begin{tabular}{|c|c|c|}
\hline $\mathrm{C}(\mathrm{m} / \mathrm{s})$ & $\mathrm{S} 1$ & $\Gamma$ \\
\hline 3940 & 1.489 & 1.99 \\
\hline
\end{tabular}

\subsection{Artificial bulk viscosity parameter}

In any numerical simulation of shock wave propagation, noise is present in the pressure results due to numerical fluctuations caused by the shock discontinuities. This noise is typically removed by introducing artificial viscosity. In LS-DYNA, two parameters, $\mathrm{Q}_{1}$ and $\mathrm{Q}_{2}$ are used to define this property, where $\mathrm{Q}_{1}$ and $\mathrm{Q}_{2}$ are the quadratic and linear coefficients. The default values for solids recommended by LS-DYNA for $\mathrm{Q}_{1}$ and $\mathrm{Q}_{2}$ are 1.5 and 0.06, respectively. However, both Selhammer [8] and Panciroli [9] recommend using 1.5 and 1 for $\mathrm{Q}_{1}$ and $\mathrm{Q}_{2}$, respectively, in the case of SPH models.

To study the effect of bulk viscosity parameter, a model with $0.01 \mathrm{~mm}$ particle spacing is created in the radial and axial directions. Material properties given in Tables 1 and 2 are used to define the model. The quadratic term, $Q_{1}$, is found to have a negligible effect on the results. Three different $\mathrm{Q}_{2}$ values are used for this study: $0.06,0.1$ and 1 . The results of the simulations are plotted in Figure 3, which indicates that using the low $\mathrm{Q}_{2}$ values results in a much stiffer response with high noise levels. It is also noted that the models with $\mathrm{Q}_{2}$ values of 0.06 and 0.1 are not able to capture the plateau. Therefore, we used the recommended $\mathrm{Q}_{2}$ value of 1 for subsequent simulations. 


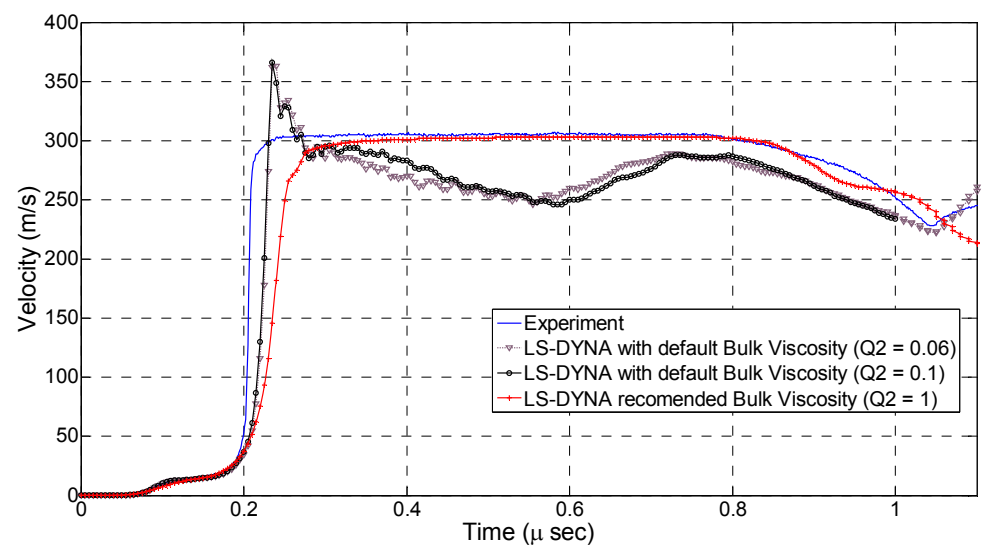

Figure 3: Effect of mesh density.

\subsection{Specific heat parameter}

During high-velocity impact events, high temperatures are produced behind the shock waves in both the impactor and target plates. As the temperature increases the yield strength of the material decreases. Therefore it is important to determine the effect of temperature on the mechanical properties of the material. In the Johnson-Cook material model, a specific heat capacity parameter is included to calculate the flow stress of the material as a function of temperature.

To study the effect of the specific heat parameter $C_{v}$, a model with $0.01 \mathrm{~mm}$ uniform particle spacing is used. Based on the results of the previous section, artificial bulk viscosity parameters, $\mathrm{Q}_{1}$ and $\mathrm{Q}_{2}$, values of 1.5 and 1 , respectively, are used in this model. Two simulations were run: one without taking temperature changes into account, and one that does using the specific heat parameter (heat transfer) of $392 \mathrm{~J} / \mathrm{kg} \mathrm{k}$ (Table 1). Figure 4 shows a comparison between the experimental results and the two models. While the two models were able to reach the same plateau as the experimental results, it is noted that the model using a specific heat parameter is able to capture the shape of the elastic precursor wave, as it better simulates the considerable increase in temperature and ductility that are associated with the impact. However, the plastic shock wave rise time in this case is much slower than that observed in the experiment.

\subsection{Particle spacing}

All previous simulations were conducted with the same particle spacing of $0.01 \mathrm{~mm}$. To study the effect of particle spacing on the problem, four models with different particle spacing were created as shown in Table 3. These models use the bulk viscosity and specific heat parameter used in earlier simulations. The results of the four cases, which can be seen in Figure 5, indicate that all 


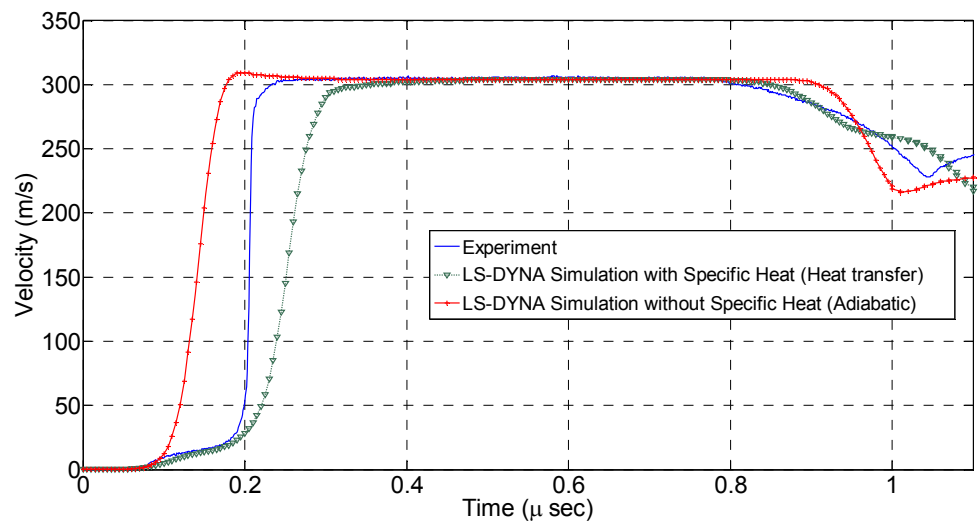

Figure 4: Effect of specific heat.

Table 3: $\quad$ Particle spacing and number of particles.

\begin{tabular}{|c|c|c|}
\hline Particle spacing $(\mathrm{mm})$ & Number of particles & $\begin{array}{c}\text { CPU run time in } \\
\text { 46 Core machine }\end{array}$ \\
\hline 0.08 & 17,064 & $\sim 5$ minutes \\
\hline 0.02 & 247,550 & $\sim 8$ hours \\
\hline 0.01 & $1,098,200$ & $\sim 12$ hours \\
\hline 0.0075 & $1,921,787$ & $\sim 17$ hours \\
\hline
\end{tabular}

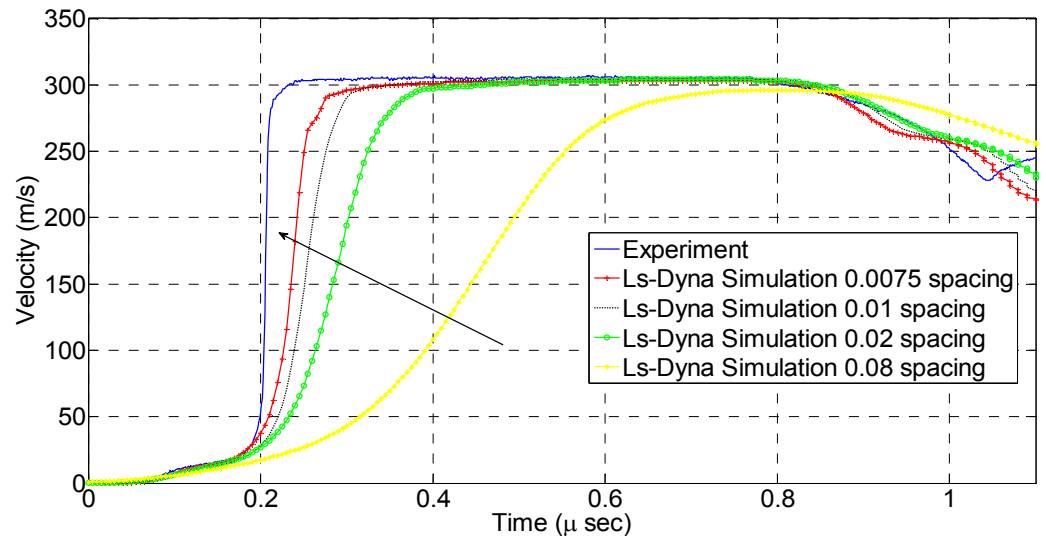

Figure 5: Mesh density.

simulations are able to capture the elastic precursor wave. However, there is a considerable variation in the rise time of the plastic shock wave. All models except for the one with the largest particle spacing are able to capture the plateau. It can be seen that as the mesh density increases, the rise time 
approaches that of the experiment. However, the problem becomes computationally expensive as the number of particles increase.

\section{Conclusion}

There are a number of parameters that play an important role in accurate modeling of one-dimensional high-velocity impact experiments using the SPH technique. The results of this work show that maintaining the bulk viscosity parameters, as recommended by other researchers, leads to a stable simulation. It is advisable to avoid modeling this problem as being adiabatic since the specific heat parameter contributes to capturing the temperature effect on the elastic precursor wave in the simulation. The results also show that increasing the mesh density improves the accuracy of the results while significantly increasing simulation time.

\section{Acknowledgements}

This manuscript has been authored by National Security Technologies, LLC, under Contract No. DE-AC52-06NA25946 with the U.S. Department of Energy and supported by the Site-Directed Research and Development Program. The United States Government retains and the publisher by accepting the article for publication, acknowledges that the United States Government retains a nonexclusive, paid-up, irrevocable, worldwide license to publish or reproduce the published form of this manuscript, or allow others to do so, for United States Government purposes.

\section{References}

[1] Jackson, K. E. \& Fuchs, Y. T., Comparison of ALE and SPH Simulations of Vertical Drop Test of a Composite Fuselage Section into Water, $10^{\text {th }}$ International LS-DYNA Users Conference, Michigan, USA, 2008

[2] Borsotti, M., Comparison of FEM and SPH for Modelling a Crushable Aircraft Arrestor Bed, Aerospace Journal, 2012

[3] Hixson, R. S., private communication, 9 September 2013, National Security Technologies, LLC, USA

[4] Barker, L. M. \& Hollenbach, R. E., Laser interferometer for measuring high velocities of any reflecting surface, J. Appl. Phys., 43(11), pp. 4669-4675, 1972

[5] Johnson, G. R. \& Cook, W. A., A Constitutive Model and Data for Metals Subjected to Large Strains, High Strain Rates and High Temperature, Symp. $7^{\text {th }}$ International Symposium on Ballistic, pp. 541-547, 1983

[6] Hallquist, J. O., LS-DYNA Keyword User's Manual, Version 971, Volume 1, Livermore Software and Technology Corporation, Livermore, CA, 2008 
[7] Marsh, S. P., LANL Shock Hugoniot Data, University of California Press, 1980

[8] Selhammer, M., Modified artificial viscosity in smooth particle hydrodynamics, Astronomy and Astrophysics, 325, pp. 857-865, 1997

[9] Panciroli, R., Hydroelastic impacts of deformable wedges, Dynamic Failure of Composite and Sandwich Structures, Solid Mechanics and Its Applications, 192, pp. 1-45, 2013 